\title{
A characterization of EM coupling in a fully electric 4-wheel drive vehicle
}

\author{
M. Iglesias ${ }^{1}$, I. Echeverria ${ }^{1}$, F. Arteche ${ }^{1}$, \\ J. Piedrafita ${ }^{1}$, A. Pradas ${ }^{1}$, F.J. Arcega ${ }^{2}$ \\ ${ }^{1}$ Instituto Tecnológico de Aragón (ITAINNOVA), \\ ${ }^{2}$ Universidad de Zaragoza, \\ Zaragoza, Spain
}

\author{
J. De Smet \\ Flanders' DRIVE \\ Lommel, Belgium
}

\begin{abstract}
Electric vehicles are complex systems in which EMC must be approached in a significantly different way to the one in conventional cars. The presence of high power supplies assembled in a very small room together with signalling, control and communications devices brings about new issues related to EM disturbances and noise coupling that must be addressed in order to ensure a good performance of the systems. To achieve this, the understanding of the way noise is generated, propagates and couples within the system is critical so as to improve the immunity of the components and, eventually, the whole car. This paper presents the results of an EMC study focused on the electromagnetic interferences that take place in a fully electric vehicle. The outcome in this work is part of an EMC approach that involves an analysis of the emissions and coupling phenomena that may cause an impact on the system safety and performance. To perform this analysis, a campaign of experimental tests has been carried out on the vehicle. This task has been performed within the E-VECTOORC project (FP7INFSO-284078), in collaboration with Jaguar Land Rover and Škoda.
\end{abstract}

Keywords-electric vehicle; EMC mapping; emissions; susceptibility; coupling

\section{INTRODUCTION}

The increasing use of electrical systems in nowadays applications is bringing about major changes in transport technologies. A relevant example is the automotive industry, where the development of the electric car is using the advantages of power electronics and electrical supplies to produce cleaner and more efficient vehicles. At the same time, the quick response and control of electrical systems allows to improve the capacities of combustion-based vehicles, which intensifies the use of electrical components [1,2].

Such an important presence of electrical components aboard the vehicles requires tighter demands in terms of electromagnetic compatibility. As a result, EMC issues are becoming a critical aspect in the design of new cars [3] where current standards, so far under the scope of the European EMC Directive (2004/104/EC), are becoming insufficient as new architectures are demanding further adaptations for limits and methodologies [4]. Besides, operating conditions, so far defined as for combustion engines, may be no longer applicable to the electric case, and so new definitions will be required. Manufacturers have developed their own requirements to meet the safety and operational demands, but the presence of fully electrical cars will require a specific EMC-oriented approach in which the electric powertrain is no longer treated as an electronic component, but as an electric installation $[5,6]$.

In fact, traction in electric cars is produced by electric motors connected to power converters which are fed by energy storage devices, making these car topologies closer to the ones in electric trains. The railways case has seen the EMC approach developed in a higher degree $[7,8]$ to ensure compatibility with signalling and communication systems in the infrastructure.

The work presented in this paper is performed within the E-VECTOORC project (FP7-INFSO-284078). This project, which involves vehicle manufacturers such as Jaguar Land Rover and Škoda, addresses the individual control of the electric motor torques of fully electric vehicles. Since control electronics are especially susceptible to EMI at the same time power converters are major sources of disturbances, EMC aspects are studied in the project. To do so, a two stage approach is followed. First, emissions and immunity issues are studied at component level, in which the interactions of the components that form the powertrain on the vehicle are analyzed separately. Some results of this study were presented in [9]. Afterwards, a study of the complete system is carried out, and the EMC mapping of the whole vehicle is produced. This offers a chance to compare and establish a correlation between the interference phenomena observed at subsystem level with the one observed with the electric vehicle, yielding a complete understanding of the EMI mechanisms that take place in the system. This information allows equipment designers to identify the most critical parts of the vehicle, set the immunity specifications and, eventually, the margin between these and the emission requirements.

This paper exposes some of the results obtained in the second stage of the study, in which the emission of EM noise in the car is mapped and correlated with measurements based on the coupling phenomena that takes place in different points 
of the car. This way, a link of emission characteristics to immunity aspects is obtained, which is a critical step to understand and improve the EMC of the incoming new electric vehicle topologies.

\section{VEHICLE UNDER TEST}

A fully electric 4-wheel drive car has been used to perform the tests. This vehicle (Fig. 1) consists of a demonstrator in which an individual control of the electric motor torques is implemented in order to improve the performance of the car and enhance the stability in different driving conditions.

The car is powered by four $42 \mathrm{~kW}$ switched reluctance motors coupled on each wheel. Each motor is driven by a power converter connected to a common $600 \mathrm{~V}$ DC bus link which supplies the energy from a battery pack located in the rear part of the vehicle. Additionally, a DC/DC power converter is used to adapt the $600 \mathrm{~V}$ battery voltage to the 12 $\mathrm{V}$ line which feeds the on board electronic equipments. The power system formed by each SR motor and power converter are similar to the ones tested in [9]. Fig. 2 shows an schematic of the vehicle topology.

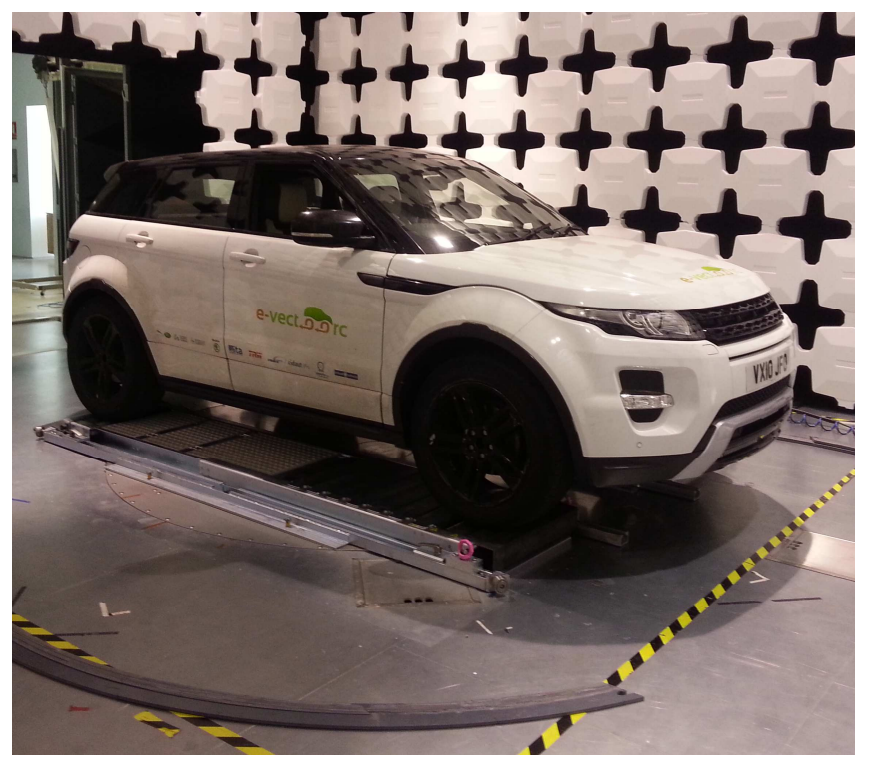

Fig. 1. Vehicle under test

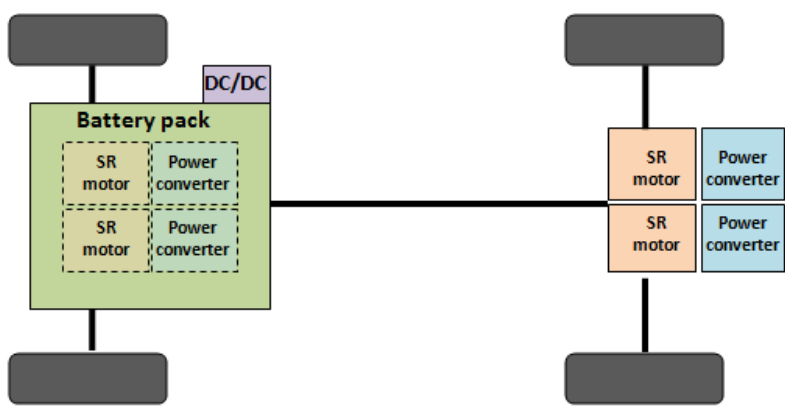

Fig. 2. Vehicle topology

\section{TESTS OVERVIEW}

In order to carry out the present study, radiated emission and coupling tests were performed. In order to achieve an optimum electromagnetic isolated environment with absorbing boundaries, the system is tested inside the ITAINNOVA $10 \times 18 \mathrm{~m}$ semi-anechoic chamber. Control and monitoring of the car is performed through a USB connection to a laptop located in the chamber control room.

Although these are non-standardized tests, some clauses in CISPR-12 [10] are used as a reference to define the vehicle conditions. Here, both stand-by and traction modes of the vehicle are tested by placing the car on top of a free roller. For the traction tests, a steady speed of $40 \mathrm{~km} / \mathrm{h}$ is selected as specified in CISPR-12.

In the following chapters, results obtained during the radiated emission and coupling tests are presented.

\section{EMISSION RESULTS}

For the emission tests (Fig. 3), the car radiated emissions are measured with three antennas covering the low and high frequency ranges.
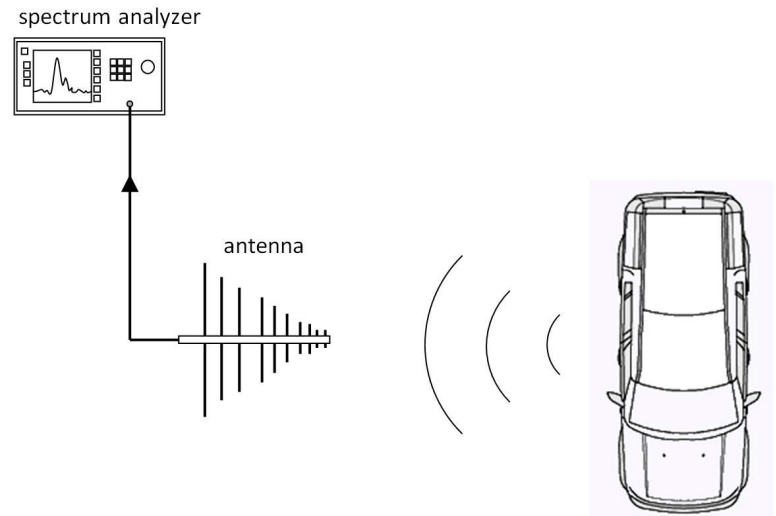

Fig. 3. EM emission test

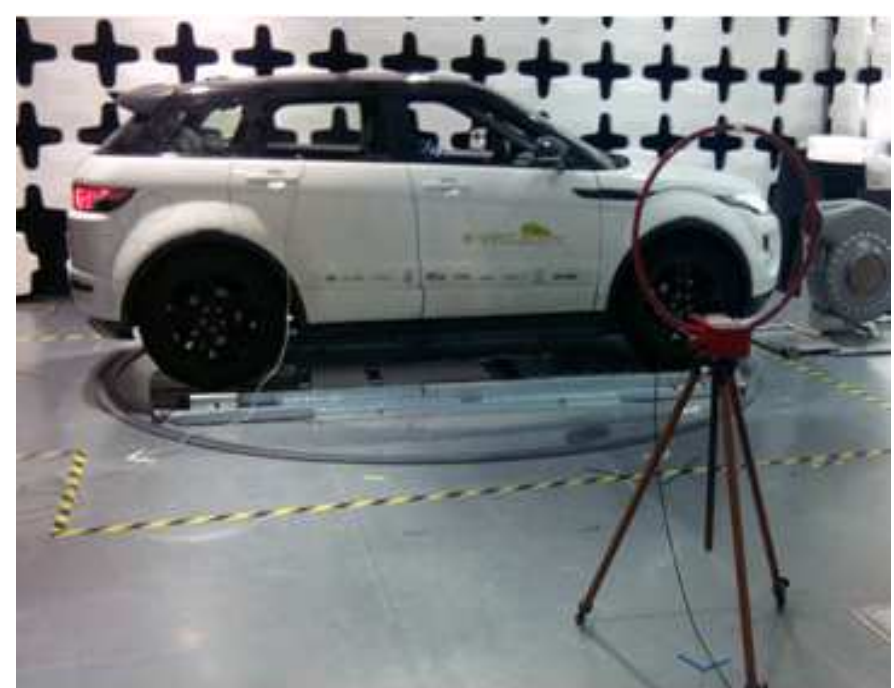

Fig. 4. Magnetic field measuring 
The antennas are placed $3 \mathrm{~m}$ from the vehicle (clause 5.2.3 of CISPR-12), and both lateral and frontal sides of the car are measured. For the magnetic field testing, a loop antenna (10 $\mathrm{kHz}-30 \mathrm{MHz}$ ) is used (Fig. 4). Electric field is measured with a dipole antenna in low frequency range $(30 \mathrm{~Hz}-50$ $\mathrm{MHz})$, while electric field in high frequency range $(30 \mathrm{MHz}-$ $2 \mathrm{GHz}$ ) is measured with a log-periodic antenna (Fig. 5) in both vertical and horizontal polarizations.

Tests are performed with the car in standby mode (motors powered but not driving traction), and traction mode (vehicle running on free coupling rolls at steady speed of $40 \mathrm{~km} / \mathrm{h}$ ), as defined in clause 5.3 of CISPR-12.

Fig. 6 shows the spectrum profile of the magnetic field emissions as measured in both front (green trace) and sideways positions (red trace). The graph also displays the measurement with the vehicle completely switched off for reference.

The measured spectra shows a rather low content of noise, with the main contribution over $10 \mathrm{MHz}$.

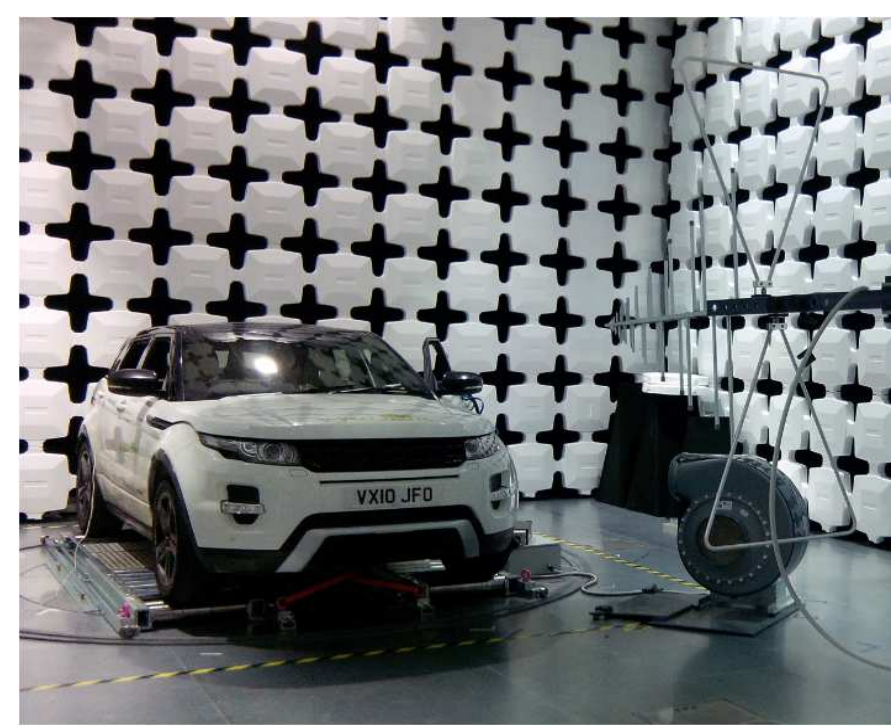

Fig. 5. Electric field measuring

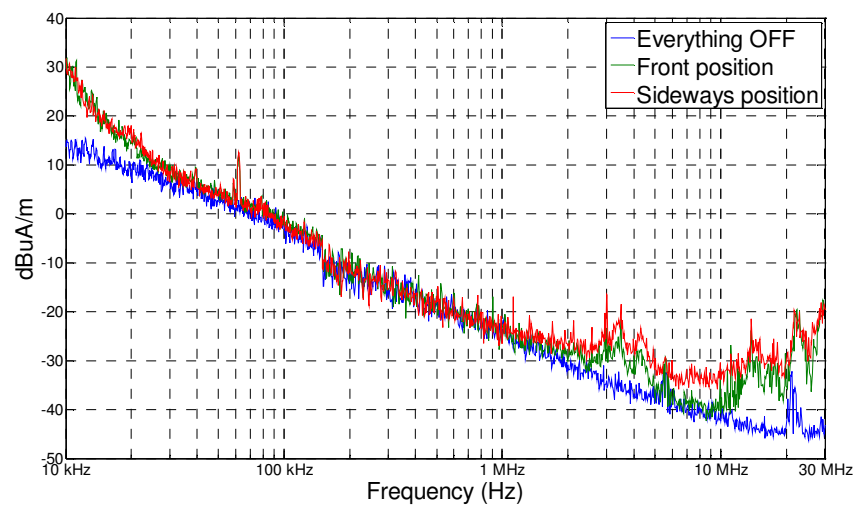

Figure 6. Magnetic field emissions

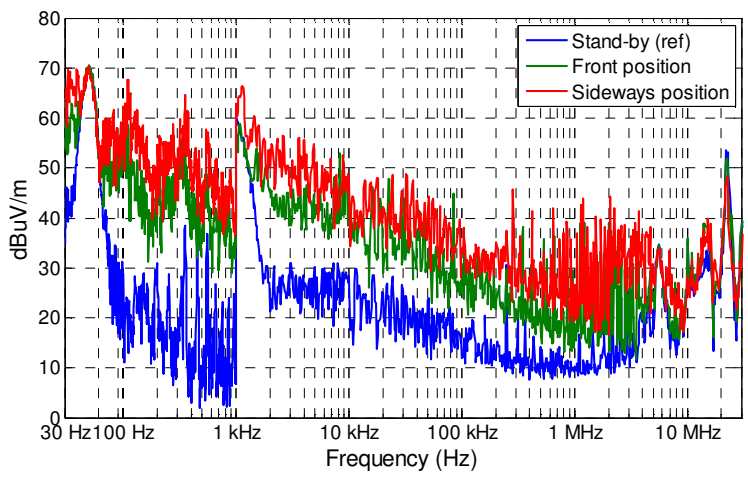

Fig. 7. Electric field emissions (low frequency)

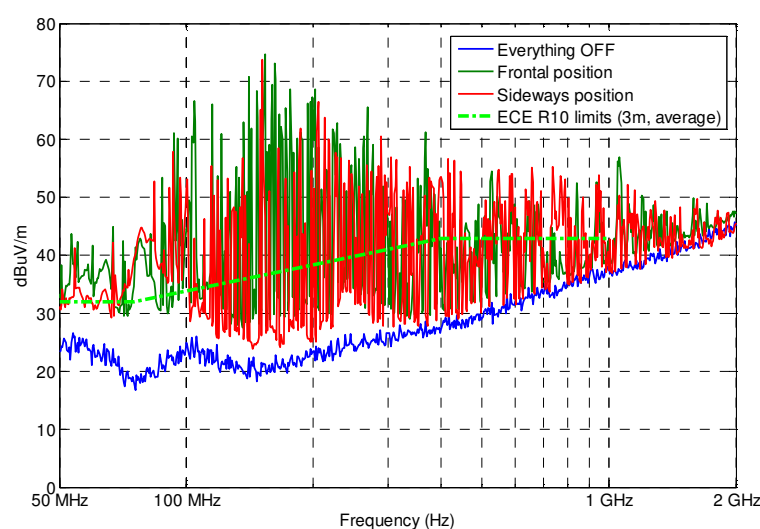

Fig. 8. Electric field emissions (high frequency) - Horizontal polarization

This involves a correct grounding and shield connection strategies in the power cables which bring about a reduction in the radiating effects of the common mode noise component, as it was shown in the emission studies performed at component level in [9]. Fig. 7 shows the electric field emission in the low frequency range, and Fig. 8 shows the electric field emission in the high frequency range for horizontal polarization (a similar profile is obtained in vertical polarization). Limits are defined in standards (ECE R10) over $30 \mathrm{MHz}$ for average and quasi-peak detector. Though the measurements have been taken with maximum detector, these limits are included in the plot as a reference.

The results show that emissions are slightly lower when the antenna is placed in frontal position below $10 \mathrm{MHz}$, however similar profiles are found in higher frequencies. It can also be observed that the harmonic content in the electric field range is significantly higher over the reference static mode when compared with the magnetic field spectra, which suggests a predominant electric behaviour of the car.

\section{RADIATED COUPLING TESTS}

The radiated coupling tests are aimed at measuring the noise that couples when an external radiated field illuminates the vehicle. This is achieved by injecting radiated noise into the car and measuring the induced conducted noise in different cables on board the vehicle (Fig. 9). 


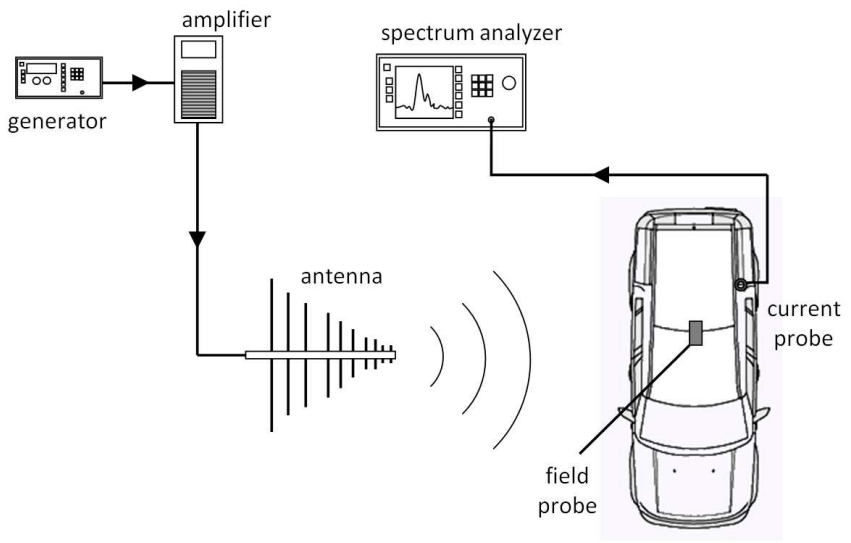

Fig. 9. EM coupling test
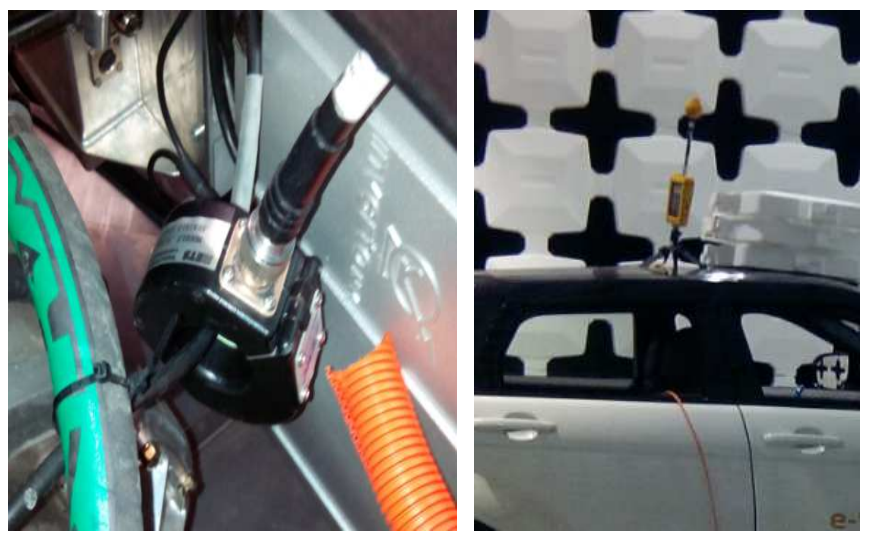

Fig. 10. Current clamp (left) and field probe (right)

A harmonic at a specific frequency is produced with a signal generator. Then it is amplified to improve the signal-tonoise ratio and injected into the antenna. The currents induced in the cables of the vehicle are measured with radio-frequency current clamps (Fig. 10, left) connected to the input of the spectrum analyzer at the generation frequency.

This point-to-point process is repeated for a set of frequencies in a way that the measuring frequency range of the current clamp (10 kHz to $400 \mathrm{MHz}$ ) is covered. To do so, three different types of antenna are used: stripline $(10 \mathrm{kHz}-30$ MHz, Fig. 11), biconical (30 MHz-150 MHz, Fig. 12) and bilog-periodic (70 MHz-3 GHz).

In order to have a reference of injected field for the coupling analysis, the field reaching the car is measured with a probe placed on top of the vehicle (Fig. 10, right). The probe is kept at the same position for every measurement, therefore the registered field values can be used as a common reference point for every current measurement.

Since coupling mechanisms are not directly related to the traction mode of the vehicle, these tests are performed only in stand-by mode.
The noise coupling of radiated disturbances on the cables is quantified and compared in a set of several points selected all over the car electric layout:

- P1: Battery output

- P2: Traction converter (front-right) input

- P3: Encoder line

- P4: Signal cables

- P5: 12V DC/DC output

- P6: Microcontroller (not visible) analog signal

In order to analyze the effect of both low and high impedance, power (P1, P2, P5) as well as signalling cables (P3, P4, P6) have been selected. Fig. 13 shows a schematic depicting the points selected for the tests.

For this analysis, a coupling transfer function [13] has been defined to measure the amount of conducted noise that is induced in a cable when exposed to an incident radiated field:

$$
T F=\frac{\text { measured current in cable }(m A)}{\text { radiated field level }(V / m)}
$$

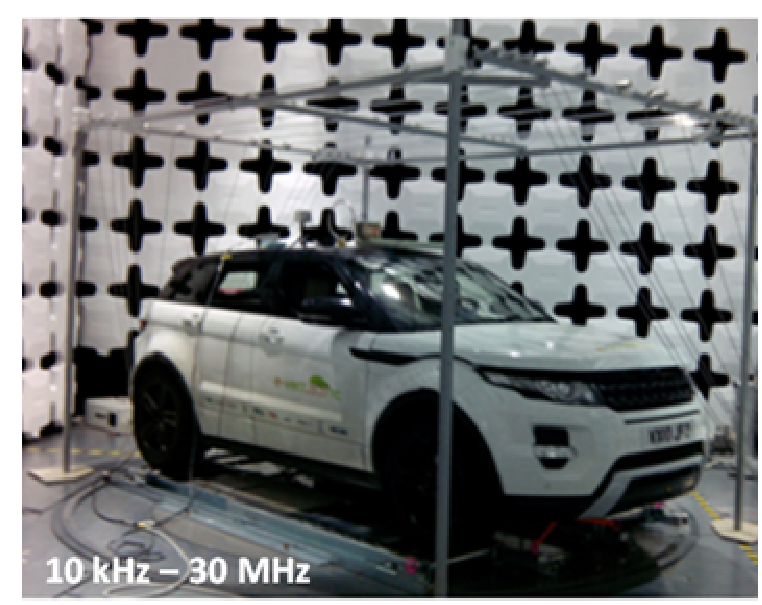

Fig. 11. Stripline antenna

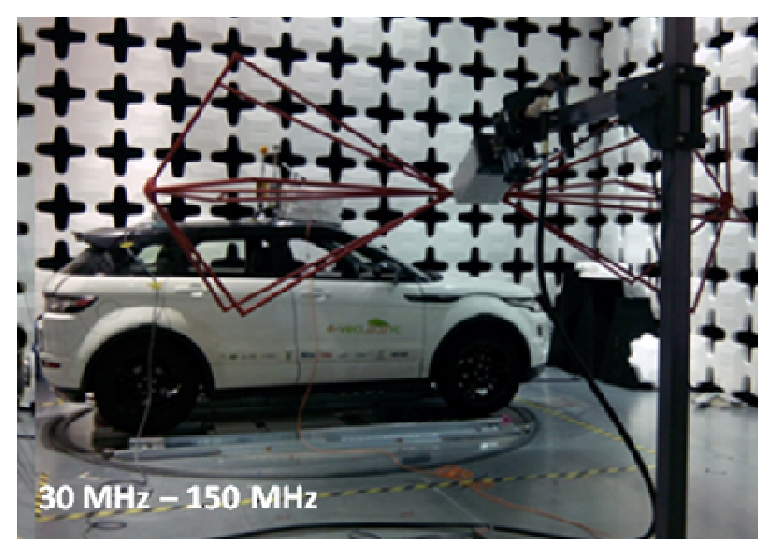

Fig. 12. Biconical antenna 
In equation (1), the measured current is obtained from the clamp (Fig. 10, left) at each one of the selected cables, whereas the radiated field level is obtained from the probe located on the roof of the vehicle (Fig. 10, right).

Fig. 14 and 15 show the transfer function computed for the different set of frequencies at each one of the tested points. Both vertical and horizontal polarizations are used for the injected radiated field.

The results show very small differences among the cables at higher frequencies, where the coupling phenomena is higher. An average representation of the coupling function over the six points is represented on Fig. 16 for both polarizations.

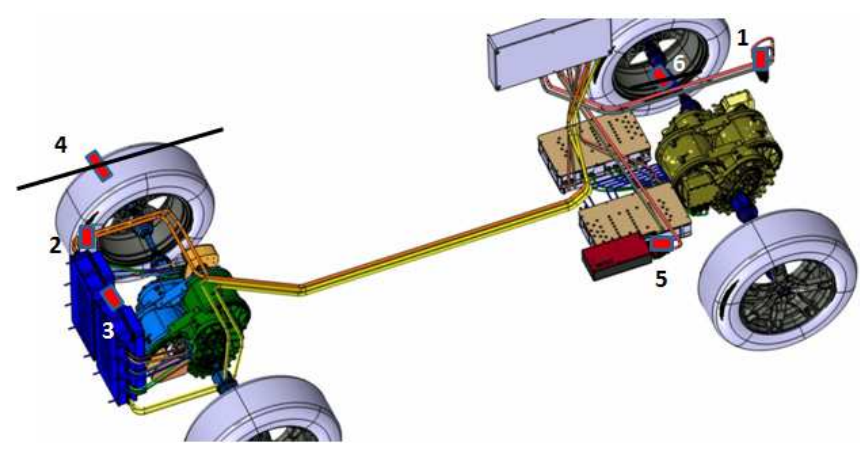

Fig. 13. Points selected for coupling tests

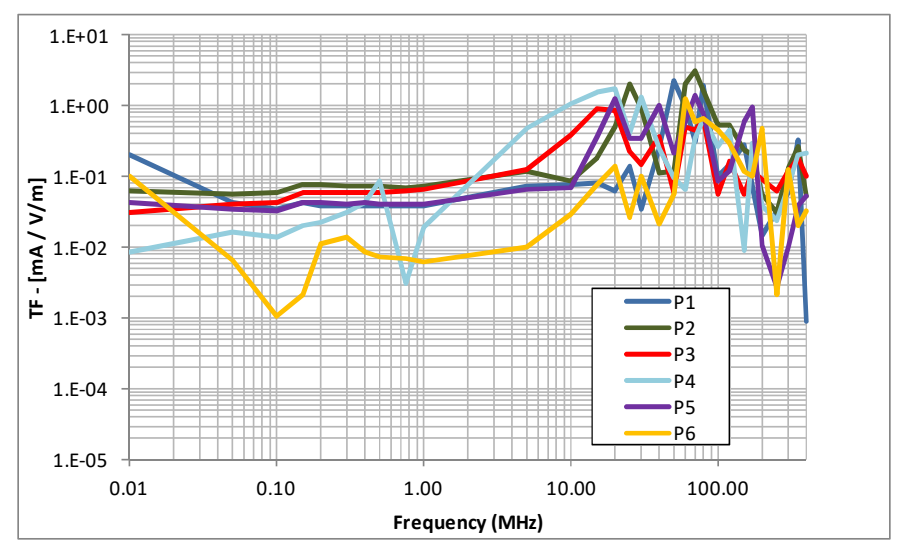

Fig. 14. Coupling transfer function for vertical polarized field

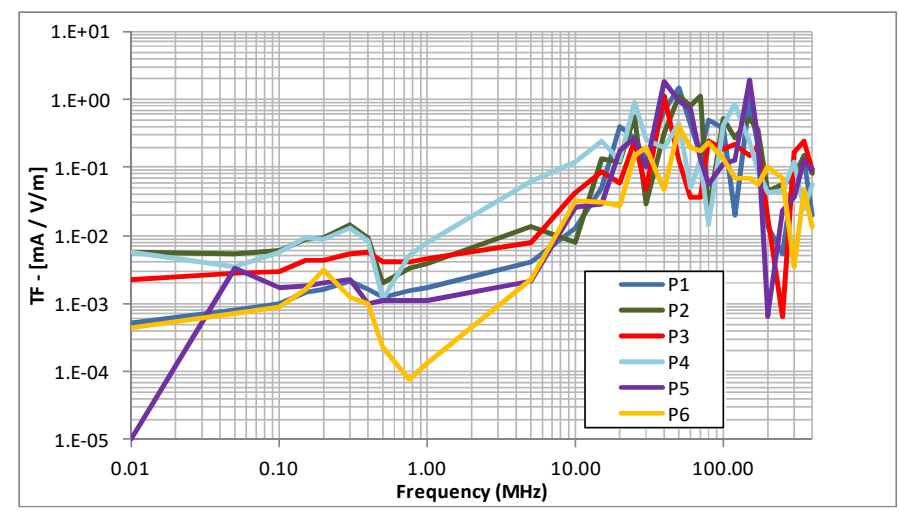

Fig. 15. Coupling transfer function for horizontal polarized field

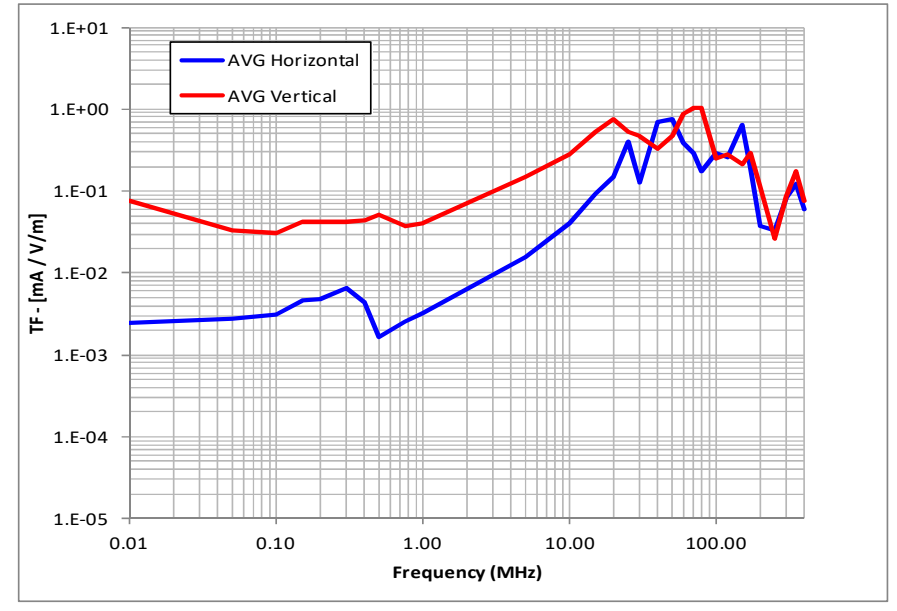

Fig. 16. Overall coupling tranfer function

Again, the graph shows a higher robustness of the system for lower frequencies, where the coupling factor is smaller. A maximum value for the transfer function is observed around $80 \mathrm{MHz}$. When comparing both polarizations, a higher efficiency in the coupling phenomena is clearly observed for the vertical case (red trace) for frequencies below $20 \mathrm{MHz}$.

\section{EMISSION AND COUPLING CORRELATION}

The measured signal spectrum in emission tests contains the narrow band noise produced by the vehicle, considered an uncontrolled noise source. On the other hand, radiated field injected into the car may involve a broadband spectrum produced by a controlled generator as well as ambient sources. However, in the case of the coupling tests the field is generated at one specific frequency in a way that a sufficiently high signal-to-noise ratio is obtained in the cable. Therefore the spectrum can be analyzed as a narrow band signal. Broadband spectra coming from other ambient sources are also avoided since the tests are performed within an isolated semi-anechoic chamber. As a result, the narrowband profile obtained in the coupling tests is compared with the narrowband profile in the emission spectra in order to link the phenomena observed in the radiated coupling tests with the emission capacity of the car.

Fig. 17 and 18 show the stand-by radiated emissions in the frequency range tested for coupling with both horizontal and vertical polarizations respectively. On the graphs, the coupling transfer function is drawn (a scale has been used in order to compare the shape of the functions). As it can be seen, the coupling factor increases along with the emissions, which illustrates that the emission efficiency of the car matches the coupling profile in the same way an antenna emits with more efficiency in the frequency range where the receiving efficiency is higher.

\section{CONCLUSIONS}

This paper has presented the results obtained during a testing campaign for an EM mapping of a fully electric car. 


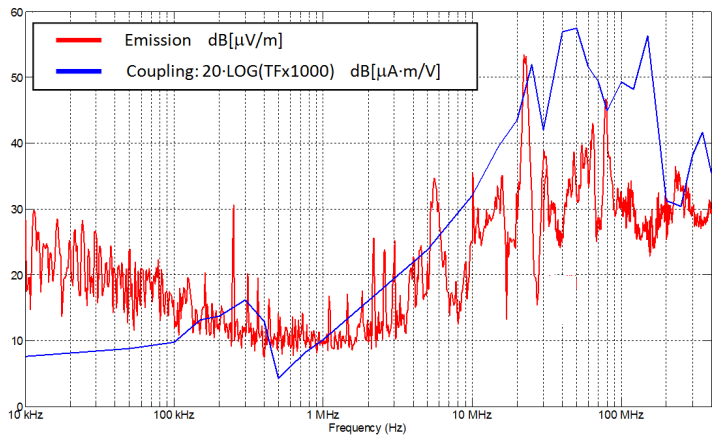

Fig. 17. Emission-coupling comparison (horizontal polarization)

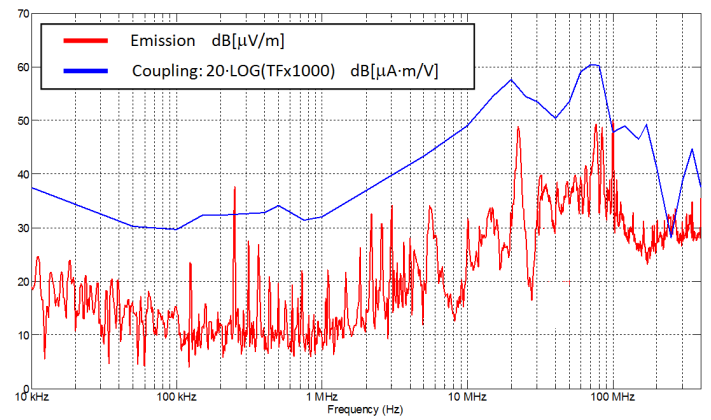

Fig. 18. Emission-coupling comparison (vertical polarization)

This has allowed to analyze both the emission profile of the vehicle as well as the coupling behaviour to radiated noise.

A set of emission tests have revealed a higher contribution of harmonics in the electric field, where the noise level is well above the reference state (with everything off). This means traction mode of the vehicle has a strong impact on the interference the car might cause (i.e. radio broadcast in the FM band), highlighting the fact the car runs as a HV electric installation. On the other hand, the magnetic emissions are much closer to the reference values. A lower emission profile of the car might be a result of a properly assembled grounding system as well as a correct connection of the shields on the cables, which contribute to confine the common mode currents that are responsible to a high degree for the magnetic emissions.

Regarding the coupling tests, the results show a similar behaviour among the tested cables, with a higher robustness in the lower frequencies. An analysis of the coupling function shows that the car is more susceptible to the vertical polarization of the incident field below $10 \mathrm{MHz}$, while both polarizations seem to present a more similar coupling efficiency above this frequency.

When comparing the coupling results with the emission spectra, similarities are detected in the $10 \mathrm{kHz}-400 \mathrm{MHz}$ profile, in which the coupling mechanism grows towards the $80 \mathrm{MHz}$ at the same time the electric field emission. This illustrates the geometrical effect of the electrical layout on the electromagnetic interactions of the car, since a dual behaviour emitter-receiver takes place.
This information, which presents the critical frequency range in which EMC issues are to be expected, can be used for designing purposes in the definition of the emission-immunity margin of the systems assembled in the car, which is of paramount importance to guarantee the electromagnetic compatibility of the whole vehicle.

\section{Acknowledgment}

The authors are grateful for the support of other members of the E-VECTOORC project consortium. The research leading to these results has received funding from the European Union's Seventh Framework Programme FP7/20072013 under grant agreement $\mathrm{n}^{\circ} 284708$. Further information can be found on the project website (www.e-vectoorc.eu).

\section{References}

[1] A. P. Asimakopoulos, B. T. Boumis, C. E. Patsias, D. A. Safacas and E. E. Mitronikas, "Experience derived from the conversion of a conventional car to a hybrid electric vehicle - Analysis of the powertrain". SPEEDAM 2010 International Symposium on Power Electronics, Electrical Drives, Automation and Motion.

[2] A. Pennycott, L. De Novellis, P. Gruber, A. Sorniotti and T. Goggia, "Enhancing the energy efficiency of fully electric vehicles via the minimization of motor power losses", 2013 IEEE International Conference on Systems, Man, and Cybernetics.

[3] S. Guttowski, S. Weber, E. Hoene, W. John, H. Reichl, "EMC Issues in cars with electric drives", 2003 IEEE International Symposium on Electromagnetic Compatibility, 777 - 782 vol.2.

[4] A. R. Ruddle, R. Armstrong, "Review of current EMC standards in relation to vehicles with electric powertrains", Proc. Of the 2013 International Symposium on Electromagnetic Compatibility (EMC Europe 2013), Brugge, Belgium, September 2-6, 2013.

[5] F. Arteche, C. Esteban, M. Iglesias, A. Gimeno, FJ. Arcega, M. Johnson and W. Cooper, "Power Network impedance effects on noise emission of DC-DC converters". Ref. JINST 7 C01045 - Journal of Instrumentation. Vol. 7, p. 8. 2012.

[6] F. Arteche, C. Esteban, I. Echeverria, M. Iglesias, C. Rivetta, I. Vila, "Global noise studies for CMS Tracker upgrade". Ref. JINST 5 C12029 - Journal of Instrumentation. Vol. 5, p. 6. 2010.

[7] Components and SubsystemsC. A. Marshman, "EMC in railways" 2008 IET Professional Development course on Electric Traction Systems

[8] EN 50121, "Railway applications - Electromagnetic compatibility", 2006.

[9] M. Iglesias, I. Echeverria, F. Arteche, J. Piedrafita, A. Pradas and J. De Clercq, , "EMC mapping of a power train for fully electric 4-wheel drive vehicle". IEEE Proceedings of EMC Europe 2014, pp.414-419.

[10] EN 55012:2008 (CISPR-12), "Vehicles, boats and internal combustion engines - Radio disturbance characteristics - Limits and methods of measurement for the protection of on-board receivers".

[11] Y. H. Lee and A. Nasiri, "Analysis and modeling of conductive EMI noise of power electronics converters in electric and hybrid electric vehicles", Twenty-Third Annual IEEE Applied Power Electronics Conference and Exposition, 2008.

[12] Y. H. Lee and A. Nasiri, "Conductive CM and DM noise analysis of power electronic converters in electric and hybrid electric vehicles", IEEE Vehicle Power and Propulsion Conference, 2007.

[13] M. Zerrer, M. Aidam, W. Koehler, S. Tenbohlen, "The Benefit of Characteristic Transfer Functions in Automotive EMC", 8th International Symposium on Antennas, Propagation and EM Theory, 2008 . 